\title{
Successful perioperative management of a patient with erythropoietin-producing uterine myoma
}

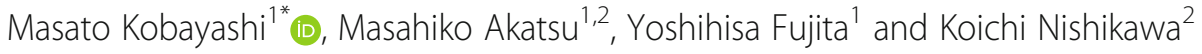

\begin{abstract}
Background: Erythropoietin-producing uterine myoma can cause various complications such as arterial or venous thrombosis and bleeding. Therefore, caution is required in the anesthetic management of affected patients.

Case presentation: A 57-year-old female was suspected to have an erythropoietin-producing uterine myoma and was scheduled to undergo an abdominal total hysterectomy and bilateral salpingo-oophorectomy. Preoperative levels of hemoglobin and erythropoietin were $21.9 \mathrm{~g} / \mathrm{dl}$ (normal 11.5-15 g/dl) and $23.2 \mathrm{IU} / \mathrm{ml}$ (normal 4.2-23.7 IU/ $\mathrm{ml})$, respectively. Preoperative phlebotomy and isovolemic hemodilution were performed to prevent arterial and venous thrombosis, following previous evidence that a hemoglobin level $<16 \mathrm{~g} / \mathrm{dl}$ reduces the occurrence of polycythemia vera-related complications. Fondaparinux $2.5 \mathrm{mg}$ was subcutaneously injected once daily after the operation, resulting in a good perioperative course without major complications.

Conclusion: Herein, we have described a successful perioperative management of a patient with erythropoietinproducing uterine myoma. Our findings in this case suggest that this combination of antithrombotic therapies can facilitate anesthetic management of patients with this disease.
\end{abstract}

Keywords: Erythropoietin-producing uterine myoma, Polycythemia, Polycythemia vera, Thrombosis, Phlebotomy, Isovolemic hemodilution, Fondaparinux

\section{Background}

Polycythemia may cause arterial and venous thrombosis [1, 2]. While some reports are available regarding the anesthetic management of polycythemia vera [3-6], to the best of our knowledge, there are no previous reports regarding the anesthetic management of erythropoietin-producing uterine myoma. Herein, we report the successful administration of general anesthesia to a patient with erythropoietin-producing uterine myoma.

\section{Case presentation}

A 57-year-old female (body height $156 \mathrm{~cm}$; body weight $64 \mathrm{~kg}$ ) was referred to our hospital due to abdominal pain caused by a large uterine myoma. Nine years prior, she was diagnosed with polycythemia and an increased erythropoietin level (Fig. 1), although she was asymptomatic. At that

\footnotetext{
* Correspondence: matokoba1107@yahoo.co.jp

${ }^{1}$ Department of Anesthesiology, Iwaki Kyoritsu General Hospital, 16 Kusehara, Mimaya-machi, Uchigo, Iwaki, Fukushima Prefecture 9738555, Japan Full list of author information is available at the end of the article
}

time, the erythropoietin level soon began decreasing slightly without medication, and thus, the follow-up was completed. However, at the time of admission to our hospital, the patient's blood test results had worsened. Although she did not report any symptoms other than abdominal pain and her activity level was not impeded, blood tests showed a relatively high level of erythropoietin and a remarkably high level of hemoglobin. Levels of hemoglobin and erythropoietin were $21.9 \mathrm{~g} / \mathrm{dl}$ (normal $11.5-15 \mathrm{~g} / \mathrm{dl}$ ) and $23.2 \mathrm{IU} / \mathrm{ml}$ (normal 4.2-23.7 IU/ml), respectively (Fig. 1). Magnetic resonance imaging revealed a large uterine myoma measuring $25 \mathrm{~cm}$ in diameter. Therefore, she was suspected to have an erythropoietin-producing uterine myoma. There were no apparent symptoms of arterial or venous thrombosis or pulmonary embolism, which were ruled out by contrast computed tomography. Platelet count, coagulation test results, fibrinogen levels, and D-dimer levels were within normal ranges.

Prior to abdominal total hysterectomy and bilateral salpingo-oophorectomy, phlebotomy was scheduled to 


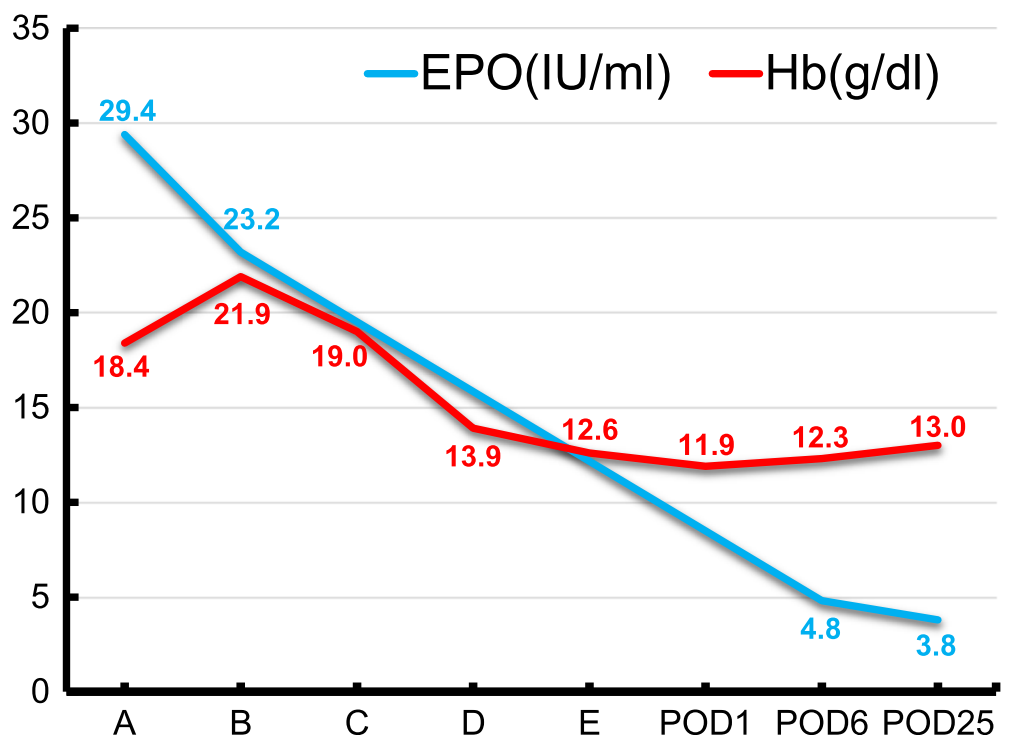

Fig. 1 Changes in hemoglobin and erythropoietin levels. A, 9 years prior to reference to our hospital; $B$, at the time of the reference to our hospital; $C$, after phlebotomy; D, shortly before the operation; $E$, shortly after the operation, EPO, erythropoietin; POD, postoperative day

treat polycythemia; this reduced the risk of arterial and venous thrombosis. The patient was phlebotomized, $300 \mathrm{ml}$ once a week, for up to 3 weeks without any complications. Despite the phlebotomy, hemoglobin levels remained high (Fig. 1); thus, isovolemic hemodilution was planned to be performed immediately following anesthesia induction.

Following placement of an epidural catheter into the epidural space at Th12/L1, general anesthesia was induced with $120 \mathrm{mg}$ propofol, $0.1 \mathrm{mg}$ fentanyl, and $50 \mathrm{mg}$ rocuronium; it was maintained with $1.5 \%$ sevoflurane, $0.25 \mu \mathrm{g} / \mathrm{kg} / \mathrm{min}$ remifentanil, and $10 \mathrm{mg}$ rocuronium per $30 \mathrm{~min}$. Electrocardiogram, bispectral index, end-tidal $\mathrm{CO}_{2}$, body temperature, and $\mathrm{SpO} 2$ were monitored during the surgery. Following induction of general anesthesia, an arterial $22 \mathrm{G}$ catheter was placed in the radial artery, from which approximately $800 \mathrm{ml}$ of blood was collected over $45 \mathrm{~min}$ while an equal amount of third-generation 6\% hydroxyethyl starch (HES) 130/0.4/ 9 was infused from a peripheral venous $18 \mathrm{G}$ catheter. As a result, the hemoglobin level dropped to $13.9 \mathrm{~g} / \mathrm{dl}$ (Fig. 1). The surgery was performed with a total blood loss of $285 \mathrm{ml}$. During surgery, the infusion mainly comprised acetic acid Ringer's solution and HES 130/0.4/9; the total infusion volume was $3600 \mathrm{ml}$. Determination of the infusion volume was based on cardiac and stroke volume indexes, measured with a FloTrac ${ }^{\mathrm{mi}} /$ Vigileo $^{\mathrm{Ts}}$ system (Edwards Lifesciences, Irvine, CA, USA; SVV $_{\text {FloTrac }}$ ). The patient's urine volume was $590 \mathrm{ml}$. At the end of the surgery, the hemoglobin level was within the normal range (Fig. 1); thus, transfusion of autologous blood was not needed. Shortly after the end of the surgery, the trachea was uneventfully extubated, and the patient was transferred to the high care unit.

On postoperative day (POD) 2, following removal of the epidural catheter, a daily subcutaneous injection of fondaparinux $2.5 \mathrm{mg}$ was initiated and continued for 5 days to prevent deep vein thrombosis and pulmonary embolism. The postoperative course was uneventful, and there were no symptoms of thrombosis or bleeding. Continuous epidural analgesia with $0.25 \%$ levobupivacaine at a rate of $5 \mathrm{ml} / \mathrm{h}$ was performed postoperatively, and the patient did not report severe pain. Hemoglobin levels remained within the normal range, and the erythropoietin level dropped dramatically (Fig. 1). Pathological examination confirmed the production of erythropoietin from the tumor cell as well as the diagnosis of erythropoietin-producing uterine myoma (Fig. 2).

\section{Discussion}

An erythropoietin-producing uterine myoma was first reported in 1953 by Thomson and Marson [7]. The prevalence of erythropoietin-producing tumors, which are mainly detected in renal cell carcinoma or hepatocellular carcinoma, is very low in secondary erythrocytosis; the prevalence of erythropoietin-producing uterine myoma is even lower, accounting for only $0.02-0.5 \%$ of all uterine myoma cases [8]. There are a few case reports of erythropoietin-producing uterine myoma investigated only from a gynecologic perspective $[9,10]$. Polycythemia vera also occurs so rarely that there is currently insufficient evidence to establish a standard anesthetic management strategy. However, a few reports regarding anesthetic management of polycythemia vera can be found $[3,4]$, 


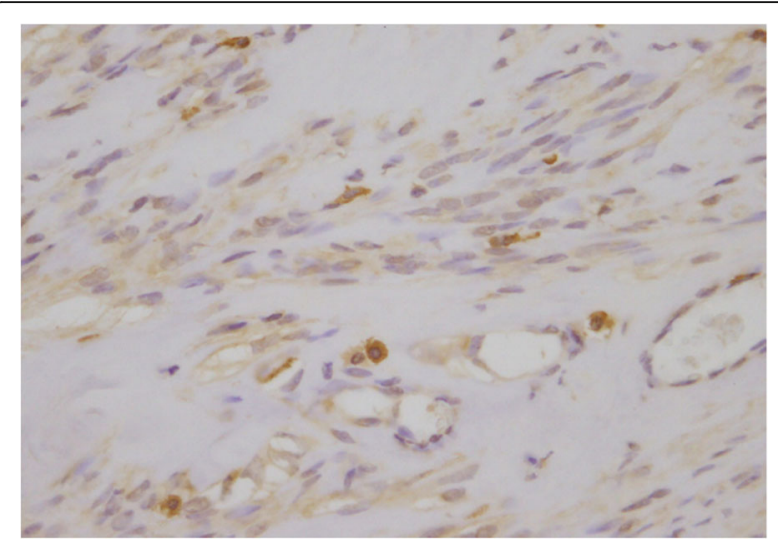

Fig. 2 Pathological examination. Immunohistochemistry showed the presence of erythropoietin in the cells of the uterine myoma. Antierythropoietin polyclonal antibody stains the cytoplasm of smooth muscle cells brown. Magnification is $\times 40$

suggesting that specific measures are required for this disease. Therefore, we took several steps to prevent arterial and venous thrombosis, in accordance with some of those reports.

Phlebotomy has been used for many years and remains an efficient approach to dilute the concentration of red blood cells with few complications. It was previously reported that the use of phlebotomy to maintain the hemoglobin and hematocrit levels below $16 \mathrm{~g} / \mathrm{dl}$ and 45\%, respectively, reduces polycythemia vera-related complications $[11,12]$. We performed phlebotomy in our patient, with a consequent decrease of the hemoglobin level. However, that decrease was insufficient to achieve the target value. With the assumption that hemoglobin and erythropoietin levels would remain low following myoma removal, we chose to proceed with the surgery, rather than repeat phlebotomy. Postponing the surgery would have extended the duration of polycythemia, increasing the risk of complications. Moreover, acute normovolemic hemodilution is helpful in cases that involve the potential for massive bleeding, as it reduces the essential amount of bleeding and avoids the risk of transfusion-related infections. In the present case, we used this method to dilute the concentration of red blood cells. It is ideal to use as thick a catheter as possible for collecting $800 \mathrm{ml}$ of blood effectively without occlusion by thrombus. Although the thickest catheter we could place was $22 \mathrm{G}$ because of the size of the artery, we were able to achieve the target hemoglobin level, as mentioned above.

The administration of low molecular weight or unfractionated heparin is also effective in reducing the risk of arterial and venous thrombosis associated with polycythemia vera, especially during the postoperative period [13]. In addition, anticoagulants including direct oral anticoagulants are recommended for the prevention of postoperative deep vein thrombosis rather than aspirin. In the present case, we administered fondaparinux, a selective inhibitor of factor $\mathrm{Xa}$, instead of heparin. Notably, once daily injection of fondaparinux $2.5 \mathrm{mg}$ has been demonstrated to reduce the relative risk of venous thrombosis in orthopedic surgeries by $50 \%$ compared to low molecular weight heparin [14]. In this case, favorable postoperative analgesia was achieved by means of epidural anesthesia. However, from the perspective of thrombosis prevention during the postoperative period, it may be reasonable to perform the ultrasound-guided transversus abdominis plane block and begin using heparin early in the postoperative period without the insertion of an epidural catheter.

Bleeding is reported as a major complication in polycythemia vera [15]. Patients with polycythemia vera can exhibit high platelet counts due to the influence of myeloproliferative disorder; bleeding tends to occur among patients with high platelet count $[6,16]$. In contrast, erythropoietin-producing uterine myoma secretes only erythropoietin; therefore, it is not associated with changes in platelet count. Accordingly, our patient's platelet count remained within the normal range. We also decided to place the epidural catheter into the epidural space for postoperative analgesia. LevGur and Levie suggested the possibility of bleeding in erythropoietin-producing uterine myoma, due to marked consumption of coagulation factors, which led to a deficiency of these factors [8]. This study further supports the use of fondaparinux. Fondaparinux is only associated with factor Xa inhibitory activity, whereas heparin also causes direct inactivation of thrombin. We believe that this characteristic of fondaparinux can lead to a lower incidence of bleeding compared with heparin. Mehta et al. reported $22 \%$ reduction in the relative risk of bleeding in the fondaparinux group compared to the unfractionated heparin group [17]. After careful consideration, the epidural catheter was successfully placed and removed without major bleeding, contributing to favorable analgesia.

\section{Conclusion}

By using phlebotomy, isovolemic hemodilution, and subcutaneous fondaparinux, successful anesthetic management of a patient with erythropoietin-producing uterine myoma was performed. This successful management suggests the possible application of polycythemia vera-specific therapies to this disease, as well as to the prevention of arterial and venous thrombosis.

\section{Abbreviations}

HES: Hydroxyethyl starch; POD: Postoperative day

\section{Acknowledgments}

We would like to thank Editage (http://www.editage.jp/) for the English language editing.

\section{Funding}

This work was supported by Grants-in-Aid for Scientific Research (C) from the Ministry of Education, Culture, Sports, Science and Technology of Japan to KN [\# 18K08820]. 


\section{Availability of data and materials}

Please contact the author for data requests.

\section{Authors' contributions}

MK drafted and revised the manuscript. MA supervised the anesthetic management and revised the manuscript. YF and KN revised the manuscript. All authors read and approved the final manuscript.

\section{Ethics approval and consent to participate}

Written informed consent was obtained from the patient, and our institutional ethical committee approved the publication of this case report.

\section{Consent for publication}

Written informed consent was obtained for the publication of this case report.

\section{Competing interests}

The authors declare that they have no competing interests.

\section{Publisher's Note}

Springer Nature remains neutral with regard to jurisdictional claims in published maps and institutional affiliations.

\section{Author details}

'Department of Anesthesiology, Iwaki Kyoritsu General Hospital, 16 Kusehara, Mimaya-machi, Uchigo, Iwaki, Fukushima Prefecture 9738555, Japan.

${ }^{2}$ Department of Disaster and Comprehensive Medicine, Fukushima Medical

University, Fukushima, Fukushima Prefecture 9601295, Japan.

Received: 30 March 2018 Accepted: 5 June 2018

Published online: 18 June 2018

\section{References}

1. Dormandy JA, Edelman JB. High blood viscosity: an aetiological factor in venous thrombosis. Br J Surg. 1973;60:187-90.

2. Lowe GD, Lee AJ, Rumley A, Price JF, Fowkes FG. Blood viscosity and risk of cardiovascular events: the Edinburgh Artery Study. Br J Haematol. 1997:96:168-73.

3. Sosis MB. Anesthesia for polycythemia vera. J Clin Anesth. 1990;2:31-4

4. Gautham NS, Arulvelan A, Manikandan S. Anesthetic management of a patient with polycythemia vera for neurosurgery. J Anesth. 2016;30:907-10

5. Mackenzie JW, Jellicoe JA. Acute upper airway obstruction. Spontaneous retropharyngeal haematoma in a patient with polycythemia rubra vera. Anesth 1986:41:57-60

6. Schmitt HJ, Becke K, Neidhardt B. Epidural anesthesia for cesarean delivery in a patient with polycythemia rubra vera and preeclampsia. Anesth Analg 2001;92:1535-7.

7. Thomason AP, Marson FG. Polycythaemia with fibroids. Lancet. 1953;262: 759-60.

8. LevGur M, Levie MD. The myomatous erythrocytosis syndrome: a review Obstet Gynecol. 1995:86:1026-30.

9. Yokoyama Y, Shinohara A, Hirokawa M, Maeda N. Erythrocytosis due to an erythropoietin-producing large uterine leiomyoma. Gynecol Obstet Investig. 2003:56:179-83.

10. Maslovsky I, Gemer O, Gefel D, Zimra Y, Lugassy G. Polycythemia as a result of ectopic erythropoietin production in benign cystic leiomyoma of uterus. Acta Obstet Gynecol Scand. 2006;85:877-88.

11. Marchioli R, Finazzi G, Specchia G, Cacciola R, Cavazzina R, Cilloni D, et al. Cardiovascular events and intensity of treatment in polycythemia vera. $\mathrm{N}$ Engl J Med. 2013;368:22-33.

12. Pearson TC, Wetherley-Mein G. Vascular occlusive episodes and venous haematocrit in primary proliferative polycythaemia. Lancet. 1978;312:1219-22

13. Ruggeri M, Rodeghiero F, Tosetto A, Castaman G, Scognamiglio F, Finazzi G, et al. Postsurgery outcomes in patients with polycythemia vera and essential thrombocythemia: a retrospective survey. Blood. 2008;111:666-71.

14. Walenga JM, Jeske WP, Samama MM, Frapaise FX, Bick RL, Fareed J, Fondaparinux: a synthetic heparin pentasaccharide as a new antithrombotic agent. Expert Opin Investig Drugs. 2002;11:397-407.

15. Wassermann LR, Gillbert HS. Surgical bleeding in polycythemia vera. Ann N Y Acad Sci. 1964;115:122-38.
16. Landolfi R, Cipriani MC, Novarese L. Thrombosis and bleeding in polycythemia vera and essential thrombocythemia: pathogenetic mechanisms and prevention. Best Pract Res Clin Haematol. 2006;19:617-33.

17. Mehta SR, Steg PG, Granger CB, Bassand JP, Faxon DP, Weitz Jl, et al. Randomized, blinded trial comparing fondaparinux with unfractionated heparin in patients undergoing contemporary percutaneous coronary intervention: Arixtra Study in percutaneous coronary intervention. A Randomized Evaluation (ASPIRE) Pilot Trial. Circulation. 2005;111:1390-7.

\section{Submit your manuscript to a SpringerOpen ${ }^{\circ}$ journal and benefit from:}

- Convenient online submission

- Rigorous peer review

- Open access: articles freely available online

- High visibility within the field

Retaining the copyright to your article 